\title{
On Lieb-Thirring Inequalities for Higher Order Operators with Critical and Subcritical Powers
}

\author{
Y. Netrusov ${ }^{1,3}$, T. Weidl ${ }^{1,2}$ \\ ${ }^{1}$ School of Mathematical Sciences, University of Sussex at Brighton, BN1 9QH Brighton Falmers, \\ UK \\ ${ }^{2}$ Royal Institute of Technology, Department of Mathematics, S-10044 Stockholm, Sweden \\ ${ }^{3}$ POMI, Fontanka 27, 191011 St. Petersburg, Russia
}

Received: 19 April 1996/Accepted: 29 April 1996

Abstract: Let $\varkappa_{i}\left(H_{l}(V)\right)$ denote the negative eigenvalues of the operator $H_{l}(V) u:=$ $(-\Delta)^{l} u-V(x) u, V \geqq 0, x \in \mathbb{R}^{d}$ on $L_{2}\left(\mathbb{R}^{d}\right)$. We prove the two-sided estimate

$$
\tilde{\mathfrak{L}}(d, l) \int_{\mathbb{R}^{d}} V(x) d x \leqq \sum_{k}\left|\varkappa_{k}\left(H_{l}(V)\right)\right|^{1-\kappa} \leqq \mathfrak{L}(d, l, 1-\kappa) \int_{\mathbb{R}^{d}} V(x) d x, \quad \kappa=d / 2 l<1 .
$$

We discuss bounds on the Riesz means $\sum_{k}\left|\varkappa_{k}\left(H_{l}(V)\right)\right|^{\mu}$ if $0<\mu<1-\kappa$.

\section{Introduction}

1.1. We consider the quadratic form

$$
\mathbf{h}_{l}(V)[u, u]:=\int_{\mathbb{R}^{d}}\left|\nabla^{l} u\right|^{2} d x-\int_{\mathbb{R}^{d}} V|u|^{2} d x, \quad 0 \leqq V \in L_{1}^{\text {loc }}\left(\mathbb{R}^{d}\right), \quad l \in \mathbb{N}_{+},
$$

defined on functions $u \in C_{0}^{\infty}\left(\mathbb{R}^{d}\right)$. If the function $V$ vanishes properly at infinity, this form can be closed. Its closure generates the self-adjoint operator

$$
H_{l}(V):=(-\Delta)^{l}-V(x)
$$

on $L_{2}\left(\mathbb{R}^{d}\right)$, the negative spectrum of which is discrete and bounded from below. Let $\left\{\varkappa_{k}\left(H_{l}(V)\right)\right\}$ stand for the non-decreasing, finite or infinite sequence of the negative eigenvalues of the operator $H_{l}(V)$.

Estimates on the negative spectrum of operators $H_{l}(V)$ in terms of the potential $V$ have been studied for many years, see e.g. [3, 6, 17, 16, 8, 14, 13, 9]. For given $d, l$ define

$$
\kappa=\kappa(d, l):=\frac{d}{2 l}, \quad v=v(d, l):=1-\frac{d}{2 l} .
$$


In [15] Lieb and Thirring proved the inequalities

$$
S_{l, \mu}(V):=\sum_{k}\left|\varkappa_{k}\left(H_{l}(V)\right)\right|^{\mu} \leqq \mathfrak{L}(d, l, \mu) \int_{\mathbb{R}^{d}} V^{\mu+\kappa}(x) d x,
$$

for potentials $0 \leqq V \in L_{\mu+\kappa}\left(\mathbb{R}^{d}\right)$ with $\mu>\max \{0, v\}$ in the case $l=1$. Their argument can easily be extended to arbitrary $l \in \mathbb{N}_{+}$, see also [10]. In $[16,8,14]$ the respective inequality was shown for $\mu=0$ if $v<0$. On the other hand it is known that (3) fails for $0 \leqq \mu<v$ if $v>0$ and for $\mu=0$ if $v=0$. In [20] the author verified (3) for $l=d=1$ and $\mu=v(1,1)=1 / 2$, where in fact the two-sided estimate

$$
\frac{1}{4} \int_{\mathbb{R}} V d x \leqq S_{1,1 / 2}(V) \leqq 1.005 \int_{\mathbb{R}} V d x, \quad d=l=1, \quad 0 \leqq V \in L_{1}(\mathbb{R}),
$$

holds, cf. [11]. In this note we prove (3) for the remaining case of a positive critical power $\mu=v(d, l)>0$ for arbitrary $d, l \in \mathbb{N}_{+}$, such that $2 l>d$. In analogy to (4) we find a two-sided estimate

$$
\tilde{\mathfrak{L}}(d, l) \int_{\mathbb{R}^{d}} V(x) d x \leqq S_{l, v}(V) \leqq \mathfrak{L}(d, l, v) \int_{\mathbb{R}^{d}} V(x) d x,
$$

which holds for all summable, non-negative potentials $0 \leqq V \in L_{1}\left(\mathbb{R}^{d}\right)$ with certain constants $0<\tilde{\mathfrak{L}}(d, l) \leqq \mathfrak{L}(d, l, v)<\infty$.

It is well-known that (3) is of sharp order in the limit of large potentials. This follows from the Weyl type asymptotical formula

$$
\begin{gathered}
S_{l, \mu}(\alpha V)=\alpha \mathfrak{Q}^{\mathrm{cl}}(d, l, \mu) \int_{\mathbb{R}^{d}} V^{\mu+\kappa} d x+o(\alpha) \quad \text { as } \alpha \rightarrow \infty, \\
\mathfrak{Q}^{\mathrm{cl}}(d, l, \mu)=\frac{\mu \Gamma(\mu) \Gamma(\kappa+1)}{2^{d} \pi^{d / 2} \Gamma\left(\frac{d}{2}+1\right) \Gamma(\kappa+\mu+1)}, \quad \mu \geqq 0,
\end{gathered}
$$

which can be obtained for sufficiently regular non-negative potentials, and which can be closed to all potentials $0 \leqq V \in L_{\mu+\kappa}\left(\mathbb{R}^{d}\right)$ if (3) holds. On the other hand for $v>0$ the operator $H_{l}(\alpha V), 0 \leqq V, 0 \neq V$ has negative spectrum for arbitrary small $\alpha>0$, and for sufficiently regular, non-negative potentials the asymptotics

$$
\begin{gathered}
S_{l, \mu}(\alpha V)=\left(\alpha \mathfrak{Q}^{0}(d, l, v) \int_{\mathbb{R}^{d}} V d x\right)^{\mu / v}+o\left(\alpha^{\mu / v}\right) \quad \text { as } \alpha \rightarrow 0, \mu>0, \\
\mathfrak{L}^{0}(d, l, v)=\frac{\pi \kappa}{\sin \pi \kappa} \mathfrak{Q}^{\mathrm{cl}}(d, l, 0),
\end{gathered}
$$

can be calculated. ${ }^{1}$ In the case of a positive critical power $\mu=v>0$ this asymptotics is of the same type as (5), and we can close (8) with $\mu / v=1$ to all potentials $0 \leqq V \in L_{1}\left(\mathbb{R}^{d}\right)$. Comparing (8) and (6) we see that a two-sided estimate can hold only in the critical case.

Naturally formula (6) agrees with the estimate (3) for supercritical powers $0<v<\mu$. However, in the scale of subcritical powers $0<\mu<v$ we find $\mu / v<$

\footnotetext{
${ }^{1}$ We include the proof of $(8)$ in the Appendix.
} 
$\mu+\kappa$, and (6) disproves (3). Hence a proper substitute of (3) for positive subcritical powers should contain two terms on the right-hand side: one of homogeneity order $\mu / v$ serving for small coupling constants, and one of Weyl type order $\mu+\kappa$, serving as $\alpha \rightarrow \infty$. In the final section of this paper we shall prove such estimates.

The authors are grateful to M. Birman, B. Davies, D. Edmunds, A. Laptev, G. Rosenblyum and D. Vassiliev for many stimulating discussions.

1.2. Notations. Below $\mathbb{Q}^{d}=\left\{x=\left(x_{1}, \ldots, x_{d}\right) \in \mathbb{R}^{d}:\left|x_{j}\right| \leqq 1 / 2, j=1, \ldots, d\right\}$. Moreover $\mathbb{N}=\{n \in \mathbb{Z}: n \geqq 0\}$, while $\mathbb{N}_{+}=\mathbb{N} \backslash\{0\}$. For a multiindex $\boldsymbol{\imath} \in \mathbb{N}^{d}$ we use the notations $|\boldsymbol{\imath}|=\sum_{j=1}^{d} l_{j}$ and $\boldsymbol{\imath} !=\prod_{j=1}^{d} l_{j}$ !. The vector $\nabla^{k}$ consists of the elements $\sqrt{\frac{k !}{\imath !}} \frac{\partial^{l}}{\partial x^{l}}$ with $|\boldsymbol{l}|=k$. Further $\Omega_{d, k}$ stands for the $\left(\begin{array}{c}k+d \\ d\end{array}\right)$-dimensional lineal of all polynomials over $\mathbb{R}^{d}$, the order of which does not exceed $k$.

Throughout the paper $\kappa$ and $v$ are defined as in (2).

Finally, if the self-adjoint operator $T$ is semi-bounded from below and its lower portion of the spectrum is discrete, then $\left\{\varkappa_{k}(T)\right\}$ denotes the non-decreasing sequence of the respective eigenvalues (according to their multiplicity).

\section{The Lieb-Thirring Inequality for Positive Critical Powers}

2.1. Main result. In this section we shall prove

Theorem 1. Assume $d, l \in \mathbb{N}_{+}$and $v=1-d / 2 l>0$. Then for all potentials $V(x)$ $\geqq 0, V \in L_{1}\left(\mathbb{R}^{d}\right)$, the inequality

$$
\tilde{\mathfrak{L}}(d, l) \int_{\mathbb{R}^{d}} V d x \leqq S_{l, v}(V) \leqq \mathfrak{L}(d, l, v) \int_{\mathbb{R}^{d}} V d x
$$

holds.

\subsection{Two covering Lemmata. We introduce}

Definition 1. Let $0 \leqq V(x) \in L_{1}\left(\mathbb{R}^{d}\right)$ have compact support. A family $\mathbf{Q}=\left\{\mathscr{Q}_{\tau}\right\}$ of cubes $\mathscr{Q}_{\tau}=x_{\tau}+a_{\tau} \mathbb{Q}^{d}, x_{\tau} \in \mathbb{R}^{d}, a_{\tau}>0$, is called a A-proper covering of supp $V$ of multiplicity $\Xi(\mathbf{Q})$, if $\operatorname{supp} V \subseteq \bigcup_{\tau} \mathscr{Q}_{\tau}$,

$$
a_{\tau}^{2 l-d} \int_{\mathscr{Q}_{\tau}} V d x=A, A>0, \quad \text { and } \quad \Xi(\mathbf{Q}):=\sup _{x \in \mathbb{R}^{d}} \sum_{\tau: x \in \operatorname{int} \mathscr{Q}_{\tau}} 1<\infty .
$$

The following result dates back to Besikovic [5]. For the convenience of the reader we give its proof and follow the argument of de Guzman [12].

Lemma 1. For each non-trivial potential $0 \leqq V \in L_{1}\left(\mathbb{R}^{d}\right)$ of compact support and any fixed $A>0$ there exists some finite A-proper covering $\mathbf{Q}(V)$ of $\operatorname{supp} V$ of multiplicity $\Xi(\mathbf{Q}(V)) \leqq 2^{d}$. 
Proof. We can assume $V \neq 0$. Then for each $x \in \mathbb{R}^{d}$ there exists a unique $a(x)>0$, such that for $\mathscr{Q}_{x}=x+a(x) \mathbb{Q}^{d}$ the equality

$$
a^{2 l-d}(x) \int_{\mathscr{Q}_{x}} V d x=A
$$

holds. The function $a: \mathbb{R}^{d} \rightarrow \mathbb{R}_{+}$is continuous and bounded from below by

$$
a(x) \geqq\left(A^{-1} \int_{\mathbb{R}^{d}} V d x\right)^{\frac{1}{d-2 l}}>0
$$

Choose $\tilde{\mathbf{Q}}=\left\{\mathscr{Q}_{x}: x \in \operatorname{supp} V\right\}$. We shall select the sought finite proper covering as an appropriate subset from $\tilde{\mathbf{Q}}$. Assume we have already chosen the points $x_{i}$, $i=1, \ldots, m$ and the respective cubes $\mathscr{Q}_{x_{i}}$. Then let $x_{m+1}$ be one of the points $x$, where the continuous function $a(x)$ achieves its maximum value on the compact set $x \in \operatorname{supp} V \backslash \bigcup_{i=1}^{m}$ int $\mathscr{Q}_{x_{i}}$. Since the interiors of the cubes $x_{i}+\frac{a\left(x_{i}\right)}{2} \mathbb{Q}^{d}$ do not intersect each other, by (11) this process stops after a finite number of iterations, and we put $\mathbf{Q}(V)=\left\{\mathscr{Q}_{x_{i}}\right\}$.

Evidently supp $V \subseteq \bigcup_{i} \mathscr{Q}_{x_{i}}$. Let us show that $\Xi(\mathbf{Q}(V)) \leqq 2^{d}$. Each of the points $x_{i}$ does not belong to the interior of any other cube than $\mathscr{2}_{x_{i}}$. Fix some point $y \in \mathbb{R}^{d}, y \neq x_{i}$. Assume $y \in \bigcap_{k=1}^{r}$ int $\mathscr{Q}_{x_{i r}}$ with $x_{i_{p}} \neq x_{i_{q}}$ for all $1 \leqq p \neq q \leqq r$ and $r>2^{d}$. Let $\vec{\imath}=\left(l_{1}, \ldots, l_{d}\right)$ denote vectors of the type $l_{k} \in\{0,1\}, k=1, \ldots, d$. Then one of the $2^{d}$ sectors $\sum_{y, \vec{\imath}}:=y+\bigotimes_{k=1}^{d}\left[0,(-1)^{t_{k}} \infty\right)$ should contain more than one of the points $x_{i_{p}}, p=1, \ldots, r$. On the other hand, if $x_{i_{p}}, x_{i_{q}} \in \sum_{y, \vec{\imath}},\left|y-x_{i_{p}}\right| \leqq$ $\left|y-x_{i_{q}}\right|, p \neq q$, and $y \in \operatorname{int} \mathscr{Q}_{x_{i p}} \cap \operatorname{int} \mathscr{Q}_{x_{i q}}$, then $x_{i_{p}} \in \operatorname{int} \mathscr{Q}_{x_{i q}}$, which contradicts the construction. Thus $r \leqq 2^{d}$.

We supplement Lemma 1 by

Lemma 2. Assume $2 l>d$. Then there exists a positive constant $\tilde{c}(d, l)$ such that from each finite A-proper covering $\mathbf{Q}(V)=\left\{\mathscr{Q}_{i}\right\}_{i=1}^{n}, \mathscr{Q}_{i}=x_{i}+a_{i} \mathbb{Q}$ of $\operatorname{supp} V$ of multiplicity $\Xi(\mathbf{Q}(V)) \leqq 2^{d}$ for a non-trivial potential $0 \leqq V \in L_{1}\left(\mathbb{R}^{d}\right)$ of compact support one can extract a subset $\mathbf{Q}^{\sharp}(V)=\left\{\mathscr{Q}_{i}\right\}_{i \in I}, I \subseteq\{1, \ldots, n\}$ with the properties

$$
\begin{gathered}
x_{i}+2 a_{i} \mathbb{Q} \cap x_{j}+2 a_{j} \mathbb{Q}=\emptyset \text { for all } i \neq j, i, j \in I, \\
\sum_{i \in I} \int_{\mathscr{Q}_{\imath}} V d x \geqq \tilde{c}(d, l) \int_{\mathbb{R}^{d}} V d x .
\end{gathered}
$$

Proof. Put $I_{0}=J_{0}=\emptyset, M_{0}=\{1, \ldots, n\}$. Assume the sets $I_{k}, J_{k}, M_{k}$ have already been constructed. If $M_{k}=\emptyset$ we abbreviate the process and take $I=I_{k}$. Otherwise choose $i_{k+1}$ such that $a_{i_{k+1}}=\min _{j \in M_{k}} a_{j}$, and take

$$
\begin{gathered}
I_{k+1}=I_{k} \cup\left\{i_{k+1}\right\}, J_{k+1}=\left\{j \in M_{k}: x_{j}+2 a_{j} \mathbb{Q} \cap x_{i_{k+1}}+2 a_{i_{k+1}} \mathbb{Q} \neq \emptyset\right\} \backslash\left\{i_{k+1}\right\}, \\
M_{k+1}=M_{k} \backslash\left(J_{k+1} \cup\left\{i_{k+1}\right\}\right) .
\end{gathered}
$$

Obviously $x_{i^{\prime}}+2 a_{i^{\prime}} \mathbb{Q} \cap x_{i^{\prime \prime}}+2 a_{i^{\prime \prime}} \mathbb{Q}=\emptyset$ for all $i^{\prime}, i^{\prime \prime} \in I$. Moreover notice that $a_{j} \geqq a_{i_{k}}$ for $j \in J_{k}$. Thus we can decompose $J_{k}$ as

$$
J_{k}=\bigcup_{m \in \mathbb{N}} J_{k}^{m}, \quad J_{k}^{m}=\left\{j \in J_{k}: 2^{m} a_{i_{k}} \leqq a_{j}<2^{m+1} a_{i_{k}}\right\} .
$$


If $j \in J_{k}^{m}$, then $\mathscr{Q}_{j} \subset x_{i_{k}}+\left(1+3 \cdot 2^{m}\right) a_{i_{k}} \mathbb{Q}$. Since $\Xi(\mathbf{Q}(V)) \leqq 2^{d}$ and vol $\mathscr{Q}_{j} \geqq 2^{m d} a_{i_{k}}^{d}$ we find

$$
\operatorname{card} J_{k}^{m} \leqq \frac{\left(1+3 \cdot 2^{m}\right)^{d} 2^{d}}{2^{m d}} \leqq 8^{d}
$$

Moreover

$$
\sum_{j \in J_{k}} \int_{\mathscr{Q}_{j}} V d x=\sum_{m \in \mathbb{N}} \sum_{j \in J_{k}^{m}} A a_{j}^{d-2 l} \leqq 8^{d} \sum_{m \in \mathbb{N}} 2^{m(d-2 l)} A a_{i_{k}}^{d-2 l}=\frac{8^{d}}{1-2^{d-2 l}} \int_{\mathscr{Q}_{l_{k}}} V d x
$$

Since $2 l>d$ we conclude

$$
\int_{\mathbb{R}^{d}} V d x \leqq \sum_{i=1}^{n} \int_{\mathscr{Q}_{l}} V d x=\sum_{k}^{\operatorname{card} I}\left(\int_{\mathscr{Q}_{l_{k}}} V d x+\sum_{j \in J_{k}} \int_{\mathscr{Q}_{J}} V d x\right) \leqq \frac{1}{\tilde{c}(d, l)} \sum_{k} \int_{\mathscr{Q}_{i_{k}}} V d x
$$

with $\tilde{c}(d, l)=\left(1-2^{d-2 l}\right) /\left(1-2^{d-2 l}+8^{d}\right)>0$.

2.3. The negative spectrum of the "Neumann" problem on the cube. In what follows put $\mathscr{Q}=a \mathbb{Q}^{d}$ for some $a>0$. Let $H_{l, \mathscr{Q}}^{N}(V)$ be the self-adjoint operator on $L_{2}(\mathbb{Q})$, corresponding to the closure of the hermitian form

$$
\mathbf{h}_{l, \mathscr{Q}}^{N}(V)[u, u]:=\int_{\mathscr{Q}}\left|\nabla^{l} u\right|^{2} d x-\int_{\mathscr{Q}} V|u|^{2} d x, \quad 0 \leqq V \in L_{1}(\mathscr{Q}), \quad u \in C^{\infty}(\mathscr{Q}) .
$$

For the negative spectrum of this operator the following standard fact holds.

Lemma 3. Assume $2 l>d, l, d \in \mathbb{N}_{+}$. Then there exists a positive finite constant $\hat{c}(d, l)$ such that, for all potentials $0 \leqq V \in L_{1}(\mathscr{Q})$ with

$$
\hat{c}(d, l) a^{2 l-d} \int_{\mathscr{Q}} V d x \leqq 1, \quad \mathscr{Q}=a \mathbb{Q}^{d}, a>0
$$

the operator $H_{l, \mathscr{Q}}^{N}(V)$ has not more than $\left(\begin{array}{c}l+d-1 \\ d\end{array}\right)$ negative eigenvalues.

Proof. By homogeneity we can take $\hat{c}(d, l)$ as the sharp constant in the inequality

$$
\begin{gathered}
|u(x)|^{2} \leqq a^{2 l-d} \hat{c}(d, l) \int_{\mathscr{Q}}\left|\nabla^{l} u(x)\right|^{2}, \\
\mathscr{Q}=a \mathbb{Q}^{d}, a>0,\left.u \in W_{2}^{l}(\mathscr{Q}) \ominus_{L_{2}(\mathscr{Q})} \Omega_{d, l-1}\right|_{\mathscr{Q}},
\end{gathered}
$$

which holds in view of the Sobolev embedding for $2 l>d$ and the theorem on equivalent norms. Because of (14) and (15) the form $\mathbf{h}_{l, 2}^{N}[u, u]$ is non-negative on $u \in C_{0}^{\infty}(\mathscr{2}) \ominus_{L_{2}(2)} \Omega_{d, l-1}$. This subspace is of codimension $\left(\begin{array}{c}l+d-1 \\ d\end{array}\right)$ in $L_{2}(\mathscr{Q})$, which by Glazmanns Lemma completes the proof. 
2.4. The Birman-Schwinger principle for $H_{l, 2}^{N}(V)$. If $2 l>d$ the resolvent of the unperturbed operator $H_{l, 2}^{N}(0)$

$$
\left(\left(H_{l, 2}^{N}(0)-\varkappa\right)^{-1} u\right)(x)=\int_{\mathscr{Q}} G_{\mathscr{Q}}(x, z, \varkappa) u(z) d z
$$

is an integral operator with a bounded continuous kernel $G_{2}(x, z, x) \in C(\mathscr{Q} \times \mathscr{2})$ for any $x<0$, see [1]. The Green function $G_{2}(x, z, x)$ obeys the homogeneity property

$$
G_{\mathscr{Q}}(x, z, \varkappa)=a^{2 l-d} G_{\mathbb{Q}}^{d}\left(a^{-1} x, a^{-1} z, a^{2 l} \varkappa\right), \quad \mathscr{Q}=a \mathbb{Q}^{d}, a>0, x<0 .
$$

From Hilberts resolvent identity one immediately concludes that

$$
\mathscr{G}_{2}(x):=\max _{x \in \mathscr{2}} G_{\mathscr{Q}}(x, x, x)
$$

is a continuous, strongly increasing function in $x<0$. Moreover

$$
\mathscr{G}_{2}(x) \rightarrow 0 \quad \text { as } x \rightarrow-\infty, \quad \mathscr{G}_{2}(x) \rightarrow+\infty \quad \text { as } x \rightarrow-0,
$$

while (16) implies

$$
\mathscr{G}_{\mathscr{Q}}(x)=a^{2 l-d} \mathscr{G}_{\mathbb{Q}}^{d}\left(a^{2 l} \chi\right), \quad \mathscr{Q}=a \mathbb{Q}^{d}, \quad a>0 .
$$

Now let $\left\{\varkappa_{k}\left(H_{l, 2}^{N}(V)\right)\right\}_{k}$ denote the non-decreasing sequence of eigenvalues of $H_{l, 2}^{N}(V)$. Consider the counting function

$$
N\left(\varkappa, H_{l, 2}^{N}(V)\right):=\sum 1:\left\{k: \varkappa_{k}\left(H_{l, 2}^{N}(V)\right)<\varkappa\right\}, \quad \varkappa<0,
$$

for the common multiplicity of the spectrum of $H_{l, 2}^{N}(V)$ below $x<0$. According to the Birman-Schwinger principle $[6,17]$ this quantity can be estimated by

$$
\begin{aligned}
N\left(\varkappa, H_{l, \mathscr{Q}}^{N}(V)\right) & \leqq \operatorname{Tr}\left\{V^{1 / 2}(x) \int_{\mathscr{Q}} G_{\mathscr{Q}}(x, z, \varkappa) V^{1 / 2}(z) \cdot d z\right\} \\
& \leqq \mathscr{G}_{\mathscr{Q}}(\varkappa) \int_{\mathscr{Q}} V(x) d x=a^{2 l-d} \mathscr{G}_{\mathbb{Q}}^{d}\left(a^{2 l} \chi\right) \int_{\mathscr{Q}} V(x) d x
\end{aligned}
$$

If we put $x=\varkappa_{1}\left(H_{l, 2}^{N}(V)\right)+0$, we find

$$
1 / \mathscr{G}_{\mathbb{Q}^{d}}\left(a^{2 l} \varkappa_{1}\left(H_{l, \mathscr{Q}}^{N}(V)\right)\right) \leqq a^{2 l-d} \int_{\mathscr{Q}} V d x .
$$

The monotone decreasing continuous function $1 / \mathscr{G}_{\mathbb{Q}}^{d}: \mathbb{R}_{-}^{0} \rightarrow \mathbb{R}_{+}^{0}$ has the monotone decreasing inverse $\mathscr{F}: \mathbb{R}_{+}^{0} \rightarrow \mathbb{R}_{-}^{0}$. Thus for the lowest eigenvalue the estimate

$$
\left|\varkappa_{1}\left(H_{l, \mathscr{Q}}^{N}(V)\right)\right|^{v} \leqq a^{d-2 l}\left|\mathscr{F}\left(a^{2 l-d} \int_{\mathscr{2}} V d x\right)\right|^{v}, \quad v=1-\frac{d}{2 l},
$$

holds. 
2.5. Proof of Theorem 1 - The estimate from above. We start with potentials $0 \leqq V \in L_{1}\left(\mathbb{R}^{d}\right)$ with compact support. Let $\mathbf{Q}(V)=\left\{\mathscr{Q}_{x_{1}}, \ldots, \mathscr{Q}_{x_{m}}\right\}$ be a $A$-proper finite covering of supp $V$ with multiplicity $\Xi(\mathbf{Q}(V)) \leqq 2^{d}$ and $A=2^{-d} / \hat{c}(d, l)$. According to (14), (19) and (10) each of the operators $H_{l, \mathscr{Q}_{x_{1}}}^{N}\left(2^{d} V\right)$ has not more than $\left(\begin{array}{c}l+d-1 \\ d\end{array}\right)$ negative eigenvalues $\varkappa_{j}\left(H_{l, \mathscr{Q}_{x_{l}}}^{N}\left(2^{d} V\right)\right)$. Put $J(i)=\left\{j: \varkappa_{j}\left(H_{l, \mathscr{Q}_{x_{l}}}^{N}\left(2^{d} V\right)\right)<0\right\}$. Then

$$
\sum_{j \in J(i)}\left|\varkappa_{j}\left(H_{l, \mathscr{Q}_{x_{l}}}^{N}\left(2^{d} V\right)\right)\right|^{v} \leqq 2^{d}\left(\begin{array}{c}
l+d-1 \\
d
\end{array}\right) \hat{c}(d, l)\left|\mathscr{F}\left(\hat{c}^{-1}(d, l)\right)\right|^{v} \int_{\mathscr{Q}_{x_{l}}} V d x .
$$

Using the variational principle and the estimate on the multiplicity of the covering it is easy to verify that

$$
\varkappa_{k}\left(H_{l}(V)\right) \geqq \chi_{k}(\hat{H}) \quad \text { for all } k: \chi_{k}\left(H_{l}(V)\right)<0, \hat{H}:=\bigoplus_{i} H_{l, \mathscr{Q}_{v_{l}}}^{N}\left(2^{d} V\right),
$$

where $\hat{H}$ acts on $\bigoplus_{i} L_{2}\left(\mathscr{Q}_{x_{i}}\right)$. The negative eigenvalues $\left\{\varkappa_{k}(\hat{H})\right\}$ of $\hat{H}$ coincides as set and in its multiplicity with the union of the sets $\left\{x_{j}\left(H_{l, \mathscr{Q}_{x_{l}}}^{N}(V)\right)<0\right\}$. For the sum of powers of negative eigenvalues of $H_{l}$ this implies

$$
\sum_{k}\left|\varkappa_{k}\left(H_{l}(V)\right)\right|^{v} \leqq \sum_{k: \varkappa_{k}(\hat{H})<0}\left|\varkappa_{k}(\hat{H})\right|^{v}=\sum_{i, j \in J(i)}\left|\varkappa_{j}\left(H_{l, \mathscr{Q}_{x_{l}}}^{N}\left(2^{d} V\right)\right)\right|^{v} \leqq \mathfrak{L}(d, l, v) \int_{\mathbb{R}^{d}} V d x
$$

The constant on the r.h.s. does not depend on the support of $V$. A standard argument allows one to close this inequality to all potentials $0 \leqq V \in L_{1}\left(\mathbb{R}^{d}\right)$.

2.6. Proof of Theorem 1 - The estimate from below. Let $\hat{\mathscr{Q}}$ be some cube in $\mathbb{R}^{d}$ and let $H_{l, \hat{Q}}^{D}(V)$ be the self-adjoint operator on $L_{2}(\hat{\mathscr{Q}})$, corresponding to the closure of the hermitian form

$$
\mathbf{h}_{l, \hat{\mathscr{Q}}}^{D}(V)[u, u]:=\int_{\hat{\mathscr{Q}}}\left|\nabla^{l} u\right|^{2} d x-\int_{\hat{\mathscr{Q}}} V|u|^{2} d x, \quad 0 \leqq V \in L_{1}(\hat{\mathscr{Q}}), \quad u \in C_{0}^{\infty}(\hat{\mathscr{Q}}) .
$$

Below $\left\{\varkappa_{k}\left(H_{l, \hat{2}}^{D}(V)\right)\right\}_{k}$ denotes the non-decreasing sequence of eigenvalues of $H_{l, \hat{2}}^{D}(V)$. Fix a function $\psi \in C_{0}^{\infty}(2 \mathbb{Q})$, such that $\psi \equiv 1$ on $\mathbb{Q}$. Put

$$
\varsigma:=\int_{2 \mathbb{Q}}\left|\nabla^{l} \psi\right|^{2} d x, \quad \vartheta:=\int_{2 \mathbb{Q}}|\psi|^{2} d x
$$

For the lowest eigenvalue of $H_{l, \hat{\mathscr{Q}}}^{D}(V)$ with $\mathscr{Q}=a \mathbb{Q}+y, \quad \hat{\mathscr{Q}}=2 a \mathbb{Q}+y, \quad a>0$, $y \in \mathbb{R}^{d}$ the variational estimate

$$
\begin{aligned}
\varkappa_{1}\left(H_{l, \hat{Q}}^{D}(V)\right) & \leqq \frac{\int_{\hat{Q}}\left|\nabla^{l} \psi\left(a^{-1}(x-y)\right)\right| d x-\int_{\hat{\mathscr{Q}}} V\left|\psi\left(a^{-1}(x-y)\right)\right|^{2} d x}{\int_{\hat{\mathscr{Q}}}\left|\psi\left(a^{-1}(x-y)\right)\right|^{2} d x} \\
& \leqq \frac{a^{d-2 l} \varsigma-\int_{\mathscr{Q}} V d x}{a^{d} \vartheta}
\end{aligned}
$$

holds. 
For potentials $0 \leqq V \in L_{1}\left(\mathbb{R}^{d}\right)$ of compact support we choose a finite $\kappa^{-1} \varsigma$ proper covering of the support of $V$, and according to Lemma 2 extract the subset

$$
\mathbf{Q}^{\sharp}(V)=\left\{\mathscr{Q}_{i}\right\}_{i \in I}, \quad \mathscr{Q}_{i}=x_{i}+a_{i} \mathbb{Q},
$$

with the properties (12), (13). From the variational principle we find that

$$
\varkappa_{k}\left(H_{l}(V)\right) \leqq \varkappa_{k}(\tilde{H}) \quad \text { for all } k: \varkappa_{k}\left(H_{l}(V)\right)<0, \tilde{H}:=\bigoplus_{i \in I} H_{l, \tilde{2}_{x_{l}}}^{D}(V),
$$

where $\tilde{H}$ acts on $L_{2}\left(\bigcup_{i \in I} \tilde{\mathscr{Q}}_{x_{i}}\right)$ with $\tilde{\mathscr{Q}}_{i}=x_{i}+2 a_{i} \mathbb{Q}$ as $i \in I$. For $\hat{\mathscr{Q}}=\tilde{\mathscr{Q}}_{i}(22)$ turns into

$$
\varkappa_{1}\left(H_{l, \tilde{\mathscr{Q}}_{l}}^{D}(V)\right) \leqq-\vartheta^{-1} v \kappa^{\kappa / v} \varsigma^{-\kappa / v}\left(\int_{\mathscr{Q}_{\imath}} V d x\right)^{1 / v} .
$$

The quantity on the r.h.s. is negative, thus $\varkappa_{1}\left(H_{l, \tilde{2}_{i}}^{D}(V)\right)<0$ and

$$
\left|\varkappa_{1}\left(H_{l, \tilde{\mathscr{Q}}_{l}}^{D}(V)\right)\right|^{v} \geqq \vartheta^{-v} v^{v} \kappa^{\kappa} \varsigma^{-\kappa} \int_{\mathscr{2}_{i}} V d x
$$

Hence from (24) and (13) we conclude

$$
\begin{aligned}
\sum_{k}\left|\varkappa_{k}\left(H_{l}(V)\right)\right|^{v} & \geqq \sum_{k: \varkappa_{k}(\tilde{H})<0}\left|\varkappa_{k}(\tilde{H})\right|^{v} \geqq \sum_{i \in I}\left|\varkappa_{1}\left(H_{l, \tilde{\mathscr{Q}}_{x_{i}}}^{D}(V)\right)\right|^{v} \\
& \geqq \vartheta^{-v} v^{v} \kappa^{\kappa} \varsigma^{-\kappa} \sum_{i \in I} \int_{\mathscr{Q}_{l}} V d x \geqq \tilde{\mathfrak{L}}(d, l) \int_{\mathbb{R}^{d}} V d x,
\end{aligned}
$$

with

$$
\tilde{\mathfrak{L}}(d, l)=\tilde{c}(d, l) \vartheta^{-v} v^{v} \kappa^{\kappa} \varsigma^{-\kappa}>0 .
$$

Closing this estimate to all $0 \leqq V \in L_{1}\left(\mathbb{R}^{d}\right)$, we complete the proof of Theorem 1 .

2.7. Positive supercritical powers. Following an argument of Lieb and Aizenman one can easily show that Theorem 1 implies

$$
S_{l, \mu}(V):=\sum_{k}\left|\varkappa_{k}\left(H_{l}(V)\right)\right|^{\mu} \leqq \mathfrak{L}(d, l, \mu) \int_{\mathbb{R}^{d}} V^{\mu+\kappa}(x) d x
$$

for all powers $\mu \geqq v>0$. As usual the condition $V \geqq 0$ in the r.h.s. of Theorem 1 can be dropped, if we substitute $V$ by $\max \{V(x), 0\}$ in the integral in the r.h.s. of (9). Then

$$
\begin{aligned}
S_{\mu, l} & =\frac{1}{B(\mu-v, v+1)} \sum_{m} \int_{0}^{\infty} \lambda^{\mu-v-1}\left(\left|\varkappa_{m}\right|-\lambda\right)_{+}^{v} d \lambda \\
& \leqq \mathfrak{L}(d, l, v) \int_{0}^{\infty} \frac{d \lambda}{\lambda} \lambda^{\mu-v} \int_{0}^{\infty} d x(V(x)-\lambda)_{+} \\
& =\frac{\mathfrak{L}(d, l, v) B(\mu-v, 2)}{B(\mu-v, v+1)} \int V^{\mu+\frac{d}{2 l}}(x) d x .
\end{aligned}
$$

Thus $\mathfrak{L}(d, l, \mu)$ is finite for all $\mu \geqq v$. 
2.8. Asymptotics for small coupling constants.

Theorem 2. Assume $\kappa=d / 2 l<1$. Then for the critical power $v=1-\kappa$ the asymptotical formula

$$
\begin{gathered}
S_{l, v}(\alpha V)=\alpha \mathfrak{Q}^{0}(d, l, v) \int_{\mathbb{R}^{d}} V d x+o(\alpha) \quad \text { as } \alpha \rightarrow 0, \\
\mathfrak{L}^{0}(d, l, v)=\frac{\pi \kappa}{2^{d} \pi^{d / 2} \Gamma\left(\frac{d}{2}+1\right) \sin \pi \kappa}
\end{gathered}
$$

holds for all potentials $0 \leqq V \in L_{1}\left(\mathbb{R}^{d}\right)$, and

$$
\sum_{k \geqq 2: \chi_{k}\left(H_{l}(\alpha V)\right)<0}\left|\chi_{k}\left(H_{l}(\alpha V)\right)\right|^{\nu}=o(\alpha) \quad \text { as } \alpha \rightarrow 0 .
$$

This theorem is based on the following two known results. For the benefit of the reader we attach the proofs of these lemmata in the Appendix.

Lemma 4. Suppose $2 l>d$ and assume the potential $0 \leqq V \in L_{1}\left(\mathbb{R}^{d}\right)$ has compact support and is not identically zero. Then there exist exactly $\left(\begin{array}{c}l+\left[\frac{d}{2}\right] \\ d\end{array}\right)$ negative eigenvalues for the operator $H_{l}(\alpha V)$ for all sufficiently small coupling constants $0<\alpha<\alpha_{0}(V)$.

Lemma 5. Suppose $2 l>d$ and $0 \leqq V \in L_{1}\left(\mathbb{R}^{d}\right)$. Then the bottom eigenvalue $\varkappa_{1}\left(H_{l}(\alpha V)\right)$ of $H_{l}(\alpha V)$ obeys the asymptotical formula

$$
\left|\varkappa_{1}\left(H_{l}(\alpha V)\right)\right|^{v}=\alpha \mathfrak{Q}^{0}(d, l, v) \int_{\mathbb{R}^{d}} V d x+o(\alpha) \quad \text { as } \alpha \rightarrow 0 .
$$

If $l+\left[\frac{d}{2}\right]>d$ and $V$ is of compact support, for the subsequent negative eigenvalues the asymptotical estimates

hold.

$$
\left|\varkappa_{j}\left(H_{l}(\alpha V)\right)\right|=o\left(\left|\varkappa_{1}\left(H_{l}(\alpha V)\right)\right|\right) \quad \text { as } \alpha \rightarrow 0, \quad j \geqq 2,
$$

Remark 1. The asymptotical formula (28) is accompanied by the well-known estimate

$$
\left|\varkappa_{1}\left(H_{l}(\alpha V)\right)\right|^{v} \leqq \alpha \mathfrak{Q}^{0}(d, l, v) \int_{\mathbb{R}^{d}} V d x,
$$

which holds for all $\alpha>0$ and $0 \leqq V \in L_{1}\left(\mathbb{R}^{d}\right)$.

Proof of Theorem 2. The formula (32) is an immediate consequence of the two previous lemmata. In view of Theorem 1 we close (26) to all potentials $V \in L_{1}\left(\mathbb{R}^{d}\right)$. Finally comparing (26) and (28), we arrive at (27).

Remark 2. Obviously

$$
\tilde{\mathfrak{I}}(d, l) \leqq \mathfrak{Q}^{\mathrm{cl}}(d, l, v)<\mathfrak{L}^{0}(d, l, v) \leqq \mathfrak{L}(d, l, v) .
$$

For the case $d=l=1$ the equality $\tilde{\mathfrak{L}}(1,1)=\mathfrak{Q}^{\mathrm{cl}}(1,1,1 / 2)=1 / 4$ is known $[20,11]$, while Lieb and Thirring conjectured $\mathfrak{L}^{0}(1,1,1 / 2)=\mathfrak{L}(1,1,1 / 2)=1 / 2$ [15]. This conjecture and the question, up to what extent

$$
\tilde{\mathfrak{L}}(d, l)=\mathfrak{L}^{\mathrm{cl}}(d, l, v) \quad \text { and } \quad \mathfrak{L}^{0}(d, l, v)=\mathfrak{L}(d, l, v)
$$

hold for general $d, l$ with $2 l>d$, remains unresolved. 
Remark 3. If $2 l>d$ for compactly supported potentials $0 \leqq V \in L_{1}\left(\mathbb{R}^{d}\right)$ the asymptotics

$$
S_{l, \mu}(\alpha V)=\left(\alpha \mathfrak{Q}^{0}(d, l, v) \int_{\mathbb{R}^{d}} V d x\right)^{\mu / v}+o\left(\alpha^{\mu / v}\right) \quad \text { as } \alpha \rightarrow 0, \mu>0
$$

holds.

\section{Lieb-Thirring Type Inequalities for Subcritical Powers}

3.1. Main result. In this section we discuss substitutes for (3), if $0<\mu<v$. Below $\mathbf{E}$ denotes the sequence of shifted unit cubes

$$
\left\{\mathscr{E}_{\vec{j}}\right\}_{\vec{j} \in \mathbb{Z}^{d}}:=\left\{\mathbb{Q}^{d}+\vec{j}\right\}_{\vec{j} \in \mathbb{Z}^{d}} .
$$

Moreover $\mathbf{F}$ stands for the sequence $\left\{\mathscr{F}_{j}\right\}_{j \in \mathbb{N}}$ with $\mathscr{F}_{1}=\mathbb{Q}^{d}$ and $\mathscr{F}_{j}:=$ $2^{j} \mathbb{Q}^{d} \backslash 2^{j-1} \mathbb{Q}^{d}, j=2,3, \ldots$. For a locally summable potential we introduce the notations $\boldsymbol{\beta}^{\mathbf{E}}(V):=\left\{\beta_{\vec{j}}^{\mathbf{E}}(V)\right\}_{\vec{j} \in \mathbb{Z}^{d}}$ and $\boldsymbol{\beta}^{\mathbf{F}}(V):=\left\{\beta_{j}^{\mathbf{F}}(V)\right\}_{j \in \mathbb{N}}$ with

$$
\beta_{\vec{j}}^{\mathbf{E}}(V):=\int_{\mathscr{E}_{\vec{j}}}|V| d x \quad \text { and } \quad \beta_{j}^{\mathbf{F}}(V):=\int_{\mathscr{F}_{j}}|V| d x
$$

Norms of such sequences have been used by Birman and Solomyak [7] to give estimates on the number of negative bound states for the operator $H_{l}(V)$ if $2 l>d$. We shall prove

Theorem 3. Assume that for $0 \leqq V \in L_{1}^{\text {loc }}\left(\mathbb{R}^{d}\right)$ the sequence $\boldsymbol{\beta}^{\mathbf{E}}(V)$ belongs to $\ell_{\mu / v}, 0<\mu<v=1-\kappa, \kappa=d / 2 l$. Then the estimate

$$
S_{l, \mu}(V) \leqq C(d, l, \mu)\left(\left\|\boldsymbol{\beta}^{\mathbf{E}}(V)\right\|_{\ell_{\mu / v}}^{\mu / v}+\left\|\boldsymbol{\beta}^{\mathbf{E}}(V)\right\|_{\ell_{\mu+\kappa}}^{\mu+\kappa}\right)
$$

holds.

Theorem 4. Assume that for $0 \leqq V \in L_{1}^{\text {loc }}\left(\mathbb{R}^{d}\right)$ the sequence $\boldsymbol{\beta}^{\mathbf{F}}\left((1+|x|)^{\sigma} V(x)\right)$ belongs to $\ell_{\mu+\kappa}, \sigma:=d(v-\mu) /(\mu+\kappa), 0<\mu<v=1-\kappa, \kappa=d / 2$ l. Put $\theta(t):=$ $t^{\mu / v}+t^{\mu+\kappa}$ for all $t \geqq 0$. Then the estimate

$$
S_{l, \mu}(V) \leqq c(d, l, \mu) \theta\left(\left\|\boldsymbol{\beta}^{\mathbf{F}}\left((1+|x|)^{\sigma} V(x)\right)\right\|_{\ell_{\mu+\kappa}}\right)
$$

holds.

3.2. Proof of Theorem 3. First we consider potentials $0 \leqq V \in L_{1}\left(\mathbb{R}^{d}\right)$ of compact support. Let $\mathbf{Q}(V)=\left\{\mathscr{Q}_{x_{l}}\right\}_{i=1}^{m}$ be a finite $A$-proper covering of $\operatorname{supp} V$, $A=2^{-d} / \hat{c}(d, l)$. Combining (21) and (20) as in the proof of Theorem 1 one finds

$$
S_{l, \mu}(V)=\sum_{k}\left|\varkappa_{k}\left(H_{l}(V)\right)\right|^{\mu} \leqq c_{3.1}(d, l, \mu) \sum_{i}\left(\int_{\mathscr{Q}_{x_{i}}} V d x\right)^{\mu / v} .
$$


Put $\mathscr{P}_{i, \vec{j}}=\mathscr{Q}_{x_{i}} \cap \mathscr{E}_{\vec{j}}$ and $I(\vec{j}):=\left\{i\right.$ : int $\left.\mathscr{P}_{i, \vec{j}} \neq \emptyset\right\}, N(\vec{j})=$ card $I(\vec{j})$. Then

$$
\begin{aligned}
\sum_{i}\left(\int_{\mathscr{Q}_{x_{i}}} V d x\right)^{\mu / v} & \leqq \sum_{i, \vec{j}}\left(\int_{\mathscr{P}_{i, \vec{j}}} V d x\right)^{\mu / v} \leqq \sum_{\vec{j}}(N(\vec{j}))^{1-\frac{\mu}{v}}\left(\sum_{i \in I(\vec{j}) \mathscr{P}_{l, \vec{j}}} V d x\right)^{\mu / v} \\
& \leqq 2^{d \mu / v} \sum_{\vec{j}}(N(\vec{j}))^{1-\frac{\mu}{v}}\left(\int_{\mathscr{E}_{\vec{J}}} V d x\right)^{\mu / v} .
\end{aligned}
$$

Next we estimate the value of $N(\vec{j})$. Therefore we split the index set $I(\vec{j})$ into

$$
I^{\prime}(\vec{j}):=\left\{i \in I(\vec{j}): \text { vol } \mathscr{Q}_{x_{i}}>1\right\}, \quad I^{\prime \prime}(\vec{j})=I(\vec{j}) \backslash I^{\prime}(\vec{j}) .
$$

If $i \in I^{\prime}(\vec{j})$ then the interior of $\mathscr{Q}_{x_{l}}$ contains at least one of the corners of $\mathscr{E}_{\vec{j}}$. Since the proper covering $\mathbf{Q}(V)$ is of a multiplicity $\Xi(\mathbf{Q}(V)) \leqq 2^{d}$, we have card $I^{\prime}(V) \leqq 2^{2 d}$. On the other hand $i \in I^{\prime \prime}(\vec{j})$ implies $\mathscr{Q}_{x_{i}} \subset \vec{j}+3 \mathbb{Q}^{d}$. Thus from $\Xi(\mathbf{Q}(V)) \leqq 2^{d}$ we obtain

$$
\sum_{i \in I^{\prime \prime}(\vec{j})} \operatorname{vol} \mathscr{Q}_{x_{i}} \leqq 6^{d}
$$

while from (10) we deduce

$$
\sum_{i \in I^{\prime \prime}(\vec{j})}\left(\operatorname{vol} \mathscr{Q}_{x_{i}}\right)^{1-\kappa^{-1}} \leqq 4^{d} \hat{c}(d, l) \int_{\vec{j}+3 \mathbb{Q}^{d}} V d x .
$$

Together (38) and (39) imply

$$
\begin{aligned}
\left(\operatorname{card} I^{\prime \prime}(\vec{j})\right)^{\kappa^{-1}} & \leqq\left(\sum_{i \in I^{\prime \prime}(\vec{j})} \operatorname{vol} \mathscr{Q}_{x_{l}}\right)^{\kappa^{-1}-1} \sum_{i \in I^{\prime \prime}(\vec{j})}\left(\operatorname{vol} \mathscr{Q}_{x_{l}}\right)^{1-\kappa^{-1}} \\
& \leqq c_{3.2}(d, l) \int_{\vec{j}+3 \mathbb{Q}^{d}} V d x
\end{aligned}
$$

thus

$$
N(\vec{j})=c_{3.3}(d, l)+c_{3.4}(d, l)\left(\int_{\vec{j}+3 \mathbb{Q}^{d}} V d x\right)^{\kappa} .
$$

Inserting this estimate into (35) and (36) we arrive at

$$
S_{l, \mu}(V) \leqq c_{3.5}(d, l, \mu)\left\|\boldsymbol{\beta}^{\mathbf{E}}(V)\right\|_{\ell_{\mu / v}}^{\mu / v}+c_{3.6}(d, l, \mu)\left(\sum_{\vec{j} \vec{j}+3 \mathbb{Q}^{d}} V d x\right)^{\mu+\kappa}
$$

which is equivalent to (33). Since the constant in this estimate does not depend on $V$, we can close the bound to all potentials $0 \leqq V$ with $\boldsymbol{\beta}(V) \in \ell_{\mu / v}$. 
3.3. Proof of Theorem 4. We consider potentials of compact support and choose a $A$-finite proper covering $\mathbf{Q}(V)$ of multiplicity $\Xi(\mathbf{Q}(V)) \leqq 2^{d}$ of the support of $V$ with $A=2^{-d} / \hat{c}(d, l)$. We put $\mathscr{P}_{i, j}=\mathscr{Q}_{x_{i}} \cap \mathscr{F}_{j}, j \in \mathbb{N}$, and $I(j):=\left\{i:\right.$ int $\left.\mathscr{P}_{i, j} \neq \emptyset\right\}$ is of cardinality $N(j)=$ card $I(j)$. In analogy to the previous proof we find

$$
S_{l, \mu}(V) \leqq c_{3.7}(d, l, \mu) \sum_{j}(N(j))^{1-\frac{\mu}{v}}\left(\int_{\mathscr{F}_{j}} V d x\right)^{\mu / v}
$$

Choose the decomposition

$$
I^{\prime}(j):=\left\{i \in I(j): \operatorname{vol} \mathscr{Q}_{x_{i}}>\max \left\{2^{-d}, 2^{d(j-3)}\right\}\right\}, \quad I^{\prime \prime}(j)=I(j) \backslash I^{\prime}(j) .
$$

If $i \in I^{\prime \prime}(j)$ then

$$
\mathscr{Q}_{x_{i}} \subset \mathscr{M}_{j}:=\bigcup_{s=\max \{1, j-1\}}^{j+1} \mathscr{F}_{s},
$$

and estimates similar to (38), (39), (40) give

$$
\operatorname{card} I^{\prime \prime}(j) \leqq c_{3.8}(d, l)\left(\operatorname{vol} \mathscr{M}_{j}\right)^{v}\left(\int_{\mathscr{M}_{j}} V d x\right)^{\kappa} \text {. }
$$

A simple geometrical argument shows, that in view of $\Xi(\mathbf{Q}(V)) \leqq 2^{d}$ the estimate

$$
\text { card } I^{\prime}(j) \leqq c_{3.9}(d)
$$

holds. Inserting $N(j)=$ card $I^{\prime}(j)+$ card $I^{\prime \prime}(j)$ with (43) and (44) into (42), we claim

$$
S_{l, \mu}(V) \leqq c_{3.10}(d, l, \mu)\left\{\sum_{j}\left(\int_{\mathscr{M}_{j}} V d x\right)^{\mu / v}+\sum_{j}\left(\operatorname{vol} \mathscr{M}_{j}\right)^{v-\mu}\left(\int_{\mathscr{M}_{j}} V d x\right)^{\mu+\kappa}\right\} .
$$

Notice that vol $\mathscr{M}_{j} \asymp(1+|x|)^{d}$ on $x \in \mathscr{M}_{j}$. Thus the second sum on the r.h.s. of (45) is bounded from above by $c_{3.11}(d, l, \mu)\left\|\boldsymbol{\beta}^{\mathbf{F}}\left((1+|x|)^{\sigma} V(x)\right)\right\|_{\ell_{\mu+\kappa}}^{\mu+\kappa}$. The first sum can be estimated by

$$
\sum_{j}\left(\int_{\mathscr{M}_{j}} V d x\right)^{\mu / v} \leqq c_{3.12}(d, l, \mu)\left\|\boldsymbol{\beta}^{\mathbf{F}}\left((1+|x|)^{\sigma} V(x)\right)\right\|_{\ell_{\mu+\kappa}}^{\mu / v}\left(\sum_{j}\left(\operatorname{vol} \mathscr{M}_{j}\right)^{-\frac{\mu \sigma q}{v d}}\right)^{q^{-1}},
$$

where we applied Hölders inequality with the powers $p=v(\mu+\kappa) / \mu>1$, $q^{-1}=1-p^{-1}$. The sum of the negative powers of vol $\mathscr{M}_{j}$ converges, which completes the proof.

3.4. Remark. From the proofs of Theorems 3 and 4 we see that in the respective bounds the term of homogeneity $\mu / v$ corresponds to large cubes $\mathscr{Q}_{x_{i}} \in \mathbf{Q}(V)$, that means areas of low density of the potential, while the term of homogeneity $\mu+\kappa$ corresponds to small cubes $\mathscr{Q}_{x_{i}} \in \mathbf{Q}(V)$, that means areas of high density of the potential. This agrees with the fact that under the conditions of these theorems we have $S_{l, \mu}(\alpha V) \asymp \alpha^{\mu / v}$ as $\alpha \rightarrow 0$, but $S_{l, \mu}(\alpha V) \asymp \alpha^{\mu+\kappa}$ as $\alpha \rightarrow \infty$. 


\section{Appendix}

In the appendix we outline the proof of Lemmata 4 and 5.

Lemma 6. Assume $2 l>d$. Put $B_{r}=\left\{x \in \mathbb{R}^{d}:|x|<r\right\}$ and let $\pi_{k}=\pi_{k}(r)$ denote the orthogonal projection in $L_{2}\left(B_{r}\right)$ onto $\left.\Omega_{d, k}\right|_{B_{r}}$. Then the inequality

$$
\left\|f-\pi_{m} f\right\|_{L_{\infty}\left(B_{r}\right)} \leqq c_{4.1}(d, l, r)\left\|\nabla^{l} f\right\|_{L_{2}\left(\mathbb{R}^{d}\right)}, \quad m=\left[l-\frac{d}{2}\right], f \in C_{0}^{\infty}\left(\mathbb{R}^{d}\right),
$$

holds.

Proof. We start from the inequalities

$$
\begin{gathered}
\left\|\nabla^{n} f\right\|_{L_{p(n)}\left(\mathbb{R}^{d}\right)} \leqq c_{4.2}(d, l, p, n)\left\|\nabla^{l} f\right\|_{L_{2}\left(\mathbb{R}^{d}\right)}, f \in C_{0}^{\infty}\left(\mathbb{R}^{d}\right), \\
2^{-1}-p^{-1}(n)=(l-n) / d, \quad n \in \mathbb{N}: m+1 \leqq n \leqq l,
\end{gathered}
$$

see e.g. [4] p. 153, Theorem 6.5.1. By the theorem on equivalent norms on $B_{r}$ we have

$$
\|g\|_{L_{2}\left(B_{r}\right)} \leqq c_{4.3}(d, l, r)\left\|\nabla^{l} g\right\|_{L_{2}\left(B_{r}\right)}=c_{4.3}(d, l, r)\left\|\nabla^{l} f\right\|_{L_{2}\left(B_{r}\right)}, \quad g:=f-\pi_{l-1} f .
$$

The Sobolev embedding $W_{2}^{l}\left(B_{r}\right) \hookrightarrow C\left(B_{r}\right)$ gives

$$
\|g\|_{L_{\infty}\left(B_{r}\right)} \leqq c_{4.4}(d, l, r)\left\|\nabla^{l} f\right\|_{L_{2}\left(B_{r}\right)} .
$$

On the other hand, applying (47) with $n=m+1$ to $f$ and $g$, we find

$$
\left\|\nabla^{n} \pi_{l-1} f\right\|_{L_{p(n)}\left(B_{r}\right)}=\left\|\nabla^{n}\left(\pi_{l-1}-\pi_{m}\right) f\right\|_{L_{p(n)}\left(B_{r}\right)} \leqq c_{4.5}(d, l, r)\left\|\nabla^{l} f\right\|_{L_{2}\left(\mathbb{R}^{d}\right)} .
$$

On the finite-dimensional lineal $\left.\left.\Omega_{d, l-1}\right|_{B_{r}} \ominus_{L_{2}\left(B_{r}\right)} \Omega_{d, m}\right|_{B_{r}}$ the norms $\left\|\nabla^{n} \cdot\right\|_{L_{p(n)}\left(B_{r}\right)}$ and $\|\cdot\|_{L_{\infty}\left(B_{r}\right)}$ are equivalent. Thus

$$
\left\|\left(\pi_{l-1}-\pi_{m}\right) f\right\|_{L_{\infty}\left(B_{r}\right)} \leqq c_{4.6}(d, l, r)\left\|\nabla^{l} f\right\|_{L_{2}\left(\mathbb{R}^{d}\right)} .
$$

From (48) and (49) we conclude (46).

4.1. Proof of Lemma 4.1. Take $r>0$ such that $\operatorname{supp} V \subset\left\{x \in \mathbb{R}^{d}:|x|<r\right\}$. From Lemma 6 we conclude

$$
\begin{aligned}
& \int_{\mathbb{R}^{d}} V\left|u-\pi_{m} u\right|^{2} d x \leqq c_{4.7}(d, l, r)\left(\int_{\mathbb{R}^{d}} V d x\right)\left\|\nabla^{l} u\right\|_{L_{2}\left(\mathbb{R}^{d}\right)}^{2}, \\
& m=\left[l-\frac{d}{2}\right], \quad u \in C_{0}^{\infty}\left(\mathbb{R}^{d}\right) .
\end{aligned}
$$

Thus the form $\mathbf{h}_{l}(\alpha V)[u, u]$ is non-negative on all

$$
u \in C_{0}^{\infty}\left(\mathbb{R}^{d}\right):\left.\pi_{m} u\right|_{B_{r}}=0,
$$

if $0<\alpha<1 /\left(c_{4.7}(d, l, r) \int V d x\right)$. The respective operator $H_{l}(\alpha V)$ has not more than rank $\pi_{m}=\left(\begin{array}{c}l+\left[\frac{d}{2}\right] \\ d\end{array}\right)$ negative eigenvalues.

2. Equip the linear space $\Omega_{d, k}$ with the norm $|p|:=\max _{\boldsymbol{l} \in \mathbb{N}^{d}:|t| \leqq k}\left|c_{\boldsymbol{l}}\right|$. Choose some function $\psi \in C^{\infty}(\mathbb{R})$, such that $\psi(t) \equiv 1$ for $t<1, \psi(t) \equiv 0$ for $t>2$ and 
$0 \leqq \psi \leqq 1$ for $1 \leqq t \leqq 2$. Define $\psi_{\varepsilon} \in C_{0}^{\infty}\left(\mathbb{R}^{d}\right)$ by $\psi_{\varepsilon}(x):=\psi(\varepsilon \ln |x|), \varepsilon>0$. A calculation shows (cf. [19], p. 123), that

$$
\int\left|\nabla^{l} \psi_{\varepsilon} p\right|^{2} d x<\varepsilon|p| M(d, l, \psi), \quad p \in \Omega_{d,\left[l-\frac{d}{2}\right]}, \quad 0<\varepsilon<1,
$$

while

$$
\int V\left|\psi_{\varepsilon} p(x)\right|^{2} d x=\int V|p(x)|^{2} d x \geqq|p| m(d, l, V),
$$

for sufficiently small $\varepsilon_{0}(V)>\varepsilon>0$ and suitable constants $0<m(d, l, V), M(d, l, \psi)$ $<\infty$. The quadratic form $\mathbf{h}_{l}(\alpha V)$ is negative on all functions $\psi_{\varepsilon} p(x) \neq 0$ from the $\left(\begin{array}{c}l+\left[\frac{d}{2}\right] \\ d\end{array}\right)$-dimensional subspace $\psi_{\varepsilon} \Omega_{d,\left[l-\frac{d}{2}\right]}$ for $0<\varepsilon<\min \left\{1, \varepsilon_{0}(V), \alpha m(d, l, V) /\right.$ $M(d, l, \psi)\}$. Thus $H_{l}(\alpha V)$ has exactly $\left(\begin{array}{c}l+\left[\frac{d}{2}\right] \\ d\end{array}\right)$ negative eigenvalues for all sufficiently small $\alpha>0$.

4.2. Proof of Lemma 5 . Let $\langle\cdot, \cdot\rangle$ denote the standard scalar product in $\mathbb{R}^{d}$. For $V \geqq 0$ we put $W(x)=\sqrt{V(x)}$ and

$$
\left(X_{\varkappa}(V) u\right)(x):=W(x) \iint_{\mathbb{R}^{d}} \frac{e^{i\langle\xi, x-y\rangle} W(y) u(y) d \xi d y}{(2 \pi)^{d}\left(|\xi|^{2 l}-x\right)}, \quad x<0 .
$$

For $V \in L_{1}\left(\mathbb{R}^{d}\right)$ and $2 l>d$ this positive integral operator acts as a HilbertSchmidt operator on $L_{2}\left(\mathbb{R}^{d}\right)$. Let $\left\{\lambda_{n}\left(X_{\varkappa}(V)\right)\right\}$ denote the non-increasing sequence of the eigenvalues of $X_{\varkappa}(V)$. Moreover $\left\{x_{n}(\alpha)\right\}:=\left\{x_{n}\left(H_{l}(\alpha V)\right)\right\}$ denotes the nondecreasing sequence of negative eigenvalues of $H_{l}(\alpha V)$. According to the BirmanSchwinger principle the identities

$$
\lambda_{k}\left(X_{\varkappa_{k}(\alpha)}(V)\right)=\alpha^{-1}, \quad k \in \mathbb{N},
$$

hold. In particular one finds

$$
\alpha^{-1}=\left\|X_{\varkappa_{1}(\alpha)}(V)\right\| \leqq \operatorname{Tr} X_{\varkappa_{1}(\alpha)}(V)=\left|\varkappa_{1}(\alpha)\right|^{-v} \mathfrak{L}^{0}(d, l, v) \int_{\mathbb{R}^{d}} V(x) d x,
$$

which turns into (30).

Assume now that $0 \leqq V(x) \in L_{1}\left(\mathbb{R}^{d}\right)$ is of compact support. We decompose the operator $X_{\varkappa}(V)$ as

$$
\begin{gathered}
X_{\varkappa}(V):=\tilde{X}_{\varkappa}(V)+\hat{X}_{\varkappa}(V)+\dot{X}_{\varkappa}(V), \\
\left(\tilde{X}_{\varkappa}(V) u\right)(x):=W(x) \int_{|\xi| \geqq 1} \int_{y \in \mathbb{R}^{d}} \frac{e^{i\langle\xi, x-y\rangle} W(y) u(y) d \xi d y}{(2 \pi)^{d}\left(|\xi|^{2 l}-x\right)}, \\
\left(\hat{X}_{\varkappa}(V) u\right)(x):=W(x) \int_{|\xi|<1} \int_{y \in \mathbb{R}^{d}} \frac{W(y) u(y) d \xi d y}{(2 \pi)^{d}\left(|\xi|^{2 l}-x\right)}, \\
\left(\dot{X}_{\varkappa}(V) u\right)(x):=W(x) \int_{|\xi|<1} \int_{y \in \mathbb{R}^{d}} \frac{\left(e^{i\langle\xi, x-y\rangle}-1\right) W(y) u(y) d \xi d y}{(2 \pi)^{d}\left(|\xi|^{2 l}-x\right)} .
\end{gathered}
$$


Evaluating the respective Hilbert-Schmidt norms we find

$$
\begin{gathered}
\left\|\tilde{X}_{\varkappa}(V)\right\| \leqq c_{4.8}(V), \\
\left\|\dot{X}_{\varkappa}(V)\right\| \leqq\left\{\begin{array}{l}
c_{4.9}(V)\left|\ln \left(e+|\varkappa|^{-1}\right)\right| \text { as } 2 l=d+1, \\
c_{4.10}(V)|\varkappa|^{\frac{d+1}{2 l}-1} \text { as } 2 l>d+1
\end{array}, \quad|\varkappa|<1 .\right.
\end{gathered}
$$

Finally we represent $\hat{X}_{\varkappa}(V)$ as

$$
\begin{gathered}
\hat{X}_{\varkappa}(V)=\hat{X}_{\varkappa}^{0}(V)+\hat{X}_{\varkappa}^{1}(V), \\
\left(\hat{X}_{\varkappa}^{0}(V) u\right)(x):=W(x) \int_{\xi \in \mathbb{R}^{d}} \int_{y \in \mathbb{R}^{d}} \frac{W(y) u(y) d \xi d y}{(2 \pi)^{d}\left(|\xi|^{2 l}-\chi\right)} .
\end{gathered}
$$

Obviously

$$
\hat{X}_{\varkappa}^{0}(V)=|\varkappa|^{\frac{d}{2 l}-1} \hat{X}_{-1}^{0}(V),
$$

and

$$
\left\|\hat{X}_{\varkappa}^{1}(V)\right\| \leqq c_{4.11}(V), \quad \varkappa<0 .
$$

We underline that the constants $c_{4.8}, \ldots, c_{4.11}$ do not depend on $\varkappa<0$.

From standard perturbation theory we conclude that the operator

$$
X_{\varkappa}(V)=|\varkappa|^{\frac{d}{2 l}-1} \hat{X}_{-1}^{0}(V)+Y_{\varkappa}(V), \quad Y_{\varkappa}(V):=\hat{H}_{\varkappa}^{1}(V)+\tilde{H}_{\varkappa}(V)+\dot{H}_{\varkappa}(V)
$$

has not more than $\operatorname{rank} X_{\varkappa}^{0}(V)=1$ eigenvalue larger than $\left\|Y_{\varkappa}(V)\right\|$, or

$$
\lambda_{k}\left(X_{\varkappa}(V)\right) \leqq c_{4.12}(V) \max \left\{|\chi|^{\frac{d+1}{2 l}-1}, \ln \left(e+|\varkappa|^{-1}\right), 1\right\} \quad \text { as }|\chi|<1, k \geqq 2 .
$$

From (30) and (51) we conclude that for compactly supported potentials $0 \leqq V$ $\in L_{1}\left(\mathbb{R}^{d}\right)$ the asymptotical estimates

$$
\left|\varkappa_{k}(\alpha)\right|=o\left(\alpha^{1 / v}\right) \quad \text { as } \alpha \rightarrow 0, k \geqq 2,
$$

hold. On the other hand for the leading eigenvalue we have

$$
|\varkappa|^{\frac{d}{2 l}-1} \lambda_{1}\left(\hat{X}_{-1}^{0}(V)\right)-\left\|Y_{\varkappa}(V)\right\| \leqq \lambda_{1}\left(X_{\varkappa}(V)\right) \leqq|\varkappa|^{\frac{d}{2 l}-1} \lambda_{1}\left(\hat{X}_{-1}^{0}(V)\right)+\left\|Y_{\varkappa}(V)\right\|,
$$

which mounts into

$\lambda_{1}\left(X_{\varkappa}(V)\right)=|x|^{\frac{d}{2 l}-1} \operatorname{Tr} \hat{X}_{-1}^{0}(V)+O\left(\max \left\{|\chi|^{\frac{d+2}{2 l}-1},\left|\ln \left(e+|\chi|^{-1}\right)\right|\right\}\right) \quad$ as $\varkappa \rightarrow-0$.

Then (30) and (51) imply

$$
\left|\chi_{1}(\alpha)\right|^{v}=\alpha \mathbf{Q}^{0}(d, l, v) \int V(x) d x+o(\alpha) \quad \text { as } \alpha \rightarrow 0 .
$$

In view of (30) we can close (52) to all potentials $0 \leqq V \in L_{1}\left(\mathbb{R}^{d}\right)$.

Remark 4. The technique of the extraction of a diverging operator of finite rank is well-known. It can be applied to the case of non-signdefined potentials and the asymptotics of the subsequent eigenvalues can also be calculated. In particular one can show that (52) remains true for compactly supported non-signdefined potentials $V \in L_{1}\left(\mathbb{R}^{d}\right)$, if only $\int V d x>0$. For the related results on the weakly coupled oneor two-dimensional Schrödinger operator we refer to [18]. 


\section{References}

1. Agmon, Sh.: On kernels, eigenvalues and eigenfunctions of operators related to elliptic problems. Comm. Pure and Appl. Math. 18, 627-663 (1965)

2. Aizenman, M., Lieb, E.: On semi-classical bounds for eigenvalues of Schrödinger operators. Phys. Lett. 66A, 427-429 (1978)

3. Bargmann, V.: On the number of bound states in a central field of force. Proc. Nat. Acad. Sci. USA 38, 961-966 (1952)

4. Bergh, J., Löfström, J.: Interpolation spaces. An Introduction. Berlin-Heidelberg-New York: Springer-Verlag 1976, $123 \mathrm{pp}$

5. Besikovitch, A.S.: A general form of the covering principle and relative differentiation of additive functions. Part I. In: Proc. Cambridge Phil. Soc. 41, 103-110 (1945) Part II. In: Proc. Cambridge Phil. Soc. 42, 1-10 (1946)

6. Birman, M.S.: The spectrum of singular boundary problems. Mat. Sb. 55, No. 2, 125-174 (1961), translated in Am. Math. Soc. Trans. (2), 53, 23-80 (1966)

7. Birman, M.Sh., Solomyak, M.Z.: Estimates for the number of negative eigenvalues of the Schrödinger operator and its generalizations. Adv. Sov. Math. 7, 1-55 (1991)

8. Cwikel, M.: Weak type estimates for singular values and the number of bound states of Schrödinger operators. Trans. AMS 224, 93-100 (1977)

9. Egorov, Yu.V., Kontrat'ev, V.A.: Estimates of the negative spectrum of an elliptic operator. Am. Math. Soc. Trans. (2), 150, 111-140 (1992)

10. Egorov, Yu.V., Kontrat'ev, V.A.: On the moments of negative eigenvalues of an elliptic operator. Math. Nachr. 174, 73-79 (1995)

11. Glaser, V., Grosse, H., Martin, A.: Bounds on the number of eigenvalues of the Schrödinger operator. Commun. Math. Phys. 59, 197-212 (1978)

12. de Guzman, M.: A covering lemma with applications to differentiability of measures and singular integral operators. Studia Math. 34, No. 3, 299-317 (1970)

13. Li, P., Yau, Sh.-T.: On the Schrödinger equation and the eigenvalue problem. Commun. Math. Phys. 88, 309-318 (1983)

14. Lieb, E.: The number of bound states of one body Schrödinger operators and the Weyl problem. Bull. Am. Math. Soc. 82, 751-753 (1976)

15. Lieb, E., Thirring, W.: Inequalities for the moments of the eigenvalues of the Schrödinger Hamiltonian and their relation to Sobolev inequalities. Studies in Math. Phys., Essays in Honor of Valentine Bargmann, Princeton, 1976

16. Rozenblum, G.V.: Distribution of the discrete spectrum of singular differential operators. Dokl. AN SSSR 202, N 5, 1012-1015 (1972), Izv. VUZov, Matematika N.1, 75-86 (1976)

17. Schwinger, Y.: On the bound states for a given potential. Proc. Nat. Acad. Sci. USA, 47, 122-129 (1961)

18. Simon, B.: The bound state of weakly coupled Schrödinger operators on one and two dimensions. Ann. Phys. 97, 279-288 (1976)

19. Solomyak, M.: A remark on the Hardy inequalities. Int. Eqs. and Op. Theory 19, 121-124 (1994)

20. Weidl, T.: On the Lieb-Thirring constants $L_{\gamma, 1}$ for $\gamma \geqq 1 / 2$. Commun. Math. Phys. 178, No. 1, 135-146 (1996)

Communicated by B. Simon 